$\begin{array}{ll}\text { Cipango } & \text { Cipango } \\ \text { Cahiers d'études japonaises }\end{array}$

$22 \mid 2015$

Du particulier et de l'universel

\title{
Le nô et l'escrime - autour du lien intime entre les familles Konparu et Yagyū
}

Noh and Fencing-The Intimate Bond between the Konparu and Yagy $\bar{u}$ Families

\section{Aya Sekoguchi}

\section{(2) OpenEdition}

1 Journals

\section{Édition électronique}

URL : https://journals.openedition.org/cipango/3126

DOI : $10.4000 /$ cipango.3126

ISSN : 2260-7706

Éditeur

INALCO

Édition imprimée

Date de publication : 1 janvier 2015

ISSN : 1164-5857

Référence électronique

Aya Sekoguchi, « Le nô et l'escrime - autour du lien intime entre les familles Konparu et Yagyū », Cipango [En ligne], 22 | 2015, mis en ligne le 12 février 2019, consulté le 10 avril 2022. URL : http:// journals.openedition.org/cipango/3126 ; DOl : https://doi.org/10.4000/cipango.3126

\section{(c) (i) (9)}

Cipango est mis à disposition selon les termes de la Licence Creative Commons Attribution - Pas d'Utilisation Commerciale 4.0 International. 


\title{
Le nô et l'escrime - autour du lien intime entre les familles Konparu et Yagyū
}

\author{
Noh and Fencing-The Intimate Bond between \\ the Konparu and Yagyü Families
}

Aya Sekoguchi

Docteur en philosophie/esthétique (EHESS), université Paris VIII

\section{Introduction}

La paix apportée par le gouvernement shogunal Tokugawa à l'époque d'Edo (1603-1868) a marqué un changement radical dans le domaine du combat. Les techniques liées aux armes, mises en œuvre jusqu'ici afin de donner la mort à l'ennemi, ont été dès lors surtout pratiquées dans le cadre de compétitions, sans mort d'homme à la clef.

L'apparition des traités du sabre, tels que le Heihō kadensho 兵法家伝書 (Livre de transmission de l'art militaire, 1632) de Yagyū Munenori 柳生宗 矩 ${ }^{1}$ (1571-1646) ou le Gorin no sho 五輪書 (Traité des cinq roues, 1645) de

1. Maître du sabre de l'école Shinkage. Fils de Yagyū Muneyoshi 柳生宗㛜 (1527-1606), qui est le fondateur de l'école Yagyū Shinkage 柳生新陰流. 
232 Cahiers d'études japonaises n²2

Miyamoto Musashi 宮本武蔵 (1584?-1645), montre que les techniques militaires se sont développées au début de cette époque en tant que voie (michi 道) ${ }^{2}$.

On a souvent mis en avant l'importance du bouddhisme zen dans la constitution des techniques guerrières comme les arts martiaux, et en particulier l'influence du moine de l'école Rinzai Takuan Sōhō 沢庵宗彭 (1573-1645) sur Yagyū Munenori. L'identité du sabre et du zen (ken zen ichinyo 剣禅一如) a été affirmée par ce moine dans le Fudōchi shinmyōroku 不動智神妙録 (La sagesse immuable, vers 1638), un traité philosophique sur l'art du sabre qui mettait notamment l'accent sur la nécessité de garder l'esprit libre de tout attachement et de toute fixation.

Certes, les maîtres d'escrime se sont inspirés de la pensée zen pour mener une réflexion sur leur art. Mais d'autres facteurs ont aussi joué un rôle considérable au moment où les techniques de combat se sont transformées en voie. Parmi ceux-ci on peut signaler l'influence du théâtre nô. Cet art et l'escrime se sont rapprochés

2. Nishiyama Matsunosuke 西山松之助, WATANABE Ichirō 渡辺一郎 et Gunji Masakatsu 郡司正勝, Kinsei geidōron 近世芸道論 (La théorie de la voie de l'art à l'époque prémoderne), Tōkyō, Iwanami shoten 岩波書店, 1972, p. 600-601. Le terme michi désignait au départ un domaine spécialisé dans l'art, puis, pendant la période médiévale, il a pris le sens de succession familiale d'un métier, en vue de la protection de la tradition face au déclin de la culture de cour. C'est pendant la période de Muromachi (1336-1573), influencée par le bouddhisme, que la notion de voie fut approfondie : les poètes Shōtetsu (1381-1459) et Shinkei (1406-1475) ont même proclamé l'identité de la poésie et du bouddhisme. Voir KonIsHi Jin.ichi 小西甚一, Michi - chüsei no rinen 道一中世の理念 (La voie - Un concept du Moyen Âge), Tōkyō, Kōdansha 講談社, 1975, p. 13 ; Ishiguro Kichijirō 石黒吉次郎, Chūsei geidōron no $s h i s \bar{o}-$ Kenkō, Zeami, Shinkei 中世芸道論の思想一兼好・世阿弥・心敬 (La pensée de la conception artistique au Moyen Âge - Kenkō, Zeami, Shinkei), Tōkyō, Kokusho kankōkai 国書刊行会, 1993, p. 15; KURASAWA Yukihiro 倉沢行洋, Geidō no tetsugaku - shükyō to gei no sōsoku 芸道の哲学一宗教と芸の相即 (La philosophie de la voie de l'art - La fusion entre la religion et l'art), Ōsaka, Tōhō shuppan 東方出版, 1983, p. 16. 
notamment au début de l'époque d'Edo à travers le lien intime entre les familles Yagyū et Konparu 金春 ${ }^{3}$, une école du nô.

Quel pourrait être alors l'apport du nô dans la constitution des arts guerriers ? On essaiera ici de présenter les deux aspects de cette contribution : l'aspect conceptuel et l'aspect pratique.

\section{Le sabre et le zen}

En 1621, Yagyū Munenori devint le maître de sabre officiel du troisième shōgun Tokugawa Iemitsu 徳川家光 (1604-1651), avec qui il entretenait un lien personnel fort ${ }^{4}$. Ce shōgun, tyrannique et emporté, suppliait Munenori de lui enseigner toujours davantage les secrets du sabre ${ }^{5}$. C'est sous la pression du shögun que Munenori eut l'occasion d'établir les principes de son école dans son grand Heihō kadensho, constitué de trois parties. La première, Shinrikyō 進履橋 ${ }^{6}$ (Le pont qui vit revenir la chaussure), autre appellation du Shinkageryū heihō no sho 新陰流兵法之書 (Livre d'art militaire de l'école Shinkage), aborde les techniques de l'école Shinkage héritées directement de Kamizumi Hidetsuna 上 泉秀綱7 (Isenokami 伊勢守，?-1577?). Les deux autres, Setsunintō 殺人刀

3. La compagnie Konparu est la plus ancienne des quatre compagnies de la province de Yamato (autour de Nara). Le treizième chef de cette école, Konparu Zenchiku 金春禅竹 (Ujinobu 氏信, 1405-vers 1470), fut le gendre et le successeur effectif de Zeami 世阿弥 (Kanze Saburō Motokiyo 観世三郎元清, 1363?-1443?).

4. À Edo, la famille Yagyū obtint une certaine prospérité grâce au statut d'instructeur militaire de la famille Tokugawa.

5. Nishiyama Matsunosuke, Watanabe Ichirō et Gunji Masakatsu, op. cit., 1972, p. 662.

6. Ce titre fait référence à l'histoire de Shih chi ou L'ancêtre aristocratique du marquis de Liu, Chang Liang, écrit par Sima Qian (145 av. J.-C. ? - 86 av. J.-C. ?). Voir YAGyū Munenori, trad. Josette Nickels-Grolier, Le Sabre de vie - Les enseignements secrets de la maison du Shôgun, Noisy-sur-École, Budo Éditions, 2005, p. 191-193.

7. Fondateur de l'école Shinkage 新陰流, une des trois plus grandes écoles d'escrime, qui égale les écoles Ittō et Shintō. Hidetsuna a transmis son enseignement à Yagyū Muneyoshi, père de Munenori. 
(Le sabre instrument de mort) et Katsuninken 活人剣 (Le sabre source de vie), décrivent les principes de l'école Yagyū Shinkage développés au cours des deux générations de Munenori et de son père.

Ce traité comprend à la fois un manuel pratique, illustré d'exemples concrets, et une réflexion sur la maîtrise mentale nécessaire à cette pratique. Pour cette dernière, l'auteur a recouru à l'aide d'un moine zen, Takuan. Né dans la province de Tajima 但馬 (dans l'actuelle préfecture de Hyōgo), issu d'une famille de samouraïs, ce moine commença par l'étude du bouddhisme de l'école 浄土宗 (École de la Terre pure), mais plus tard se convertit à l'école Rinzai 臨済宗 du bouddhisme zen. En 1608, à seulement 35 ans, il devint le supérieur du Daitoku-ji 大徳寺à Kyōto, temple de l'école Rinzai.

Néanmoins, en 1629, après avoir désavoué en public la politique du gouvernement Tokugawa sur les droits de succession ecclésiastiques, il fut exilé à Dewa 出羽, une lointaine province au nord du pays. Pourtant, grâce à l'amnistie consécutive au décès du shögun Tokugawa Hidetada en 1632, il fut autorisé à rentrer à Edo, et sa relation personnelle avec Munenori put commencer.

Takuan a laissé à Munenori le traité Fudōchi shinmyōrokü, premier ouvrage où les arcanes de l'escrime sont expliqués au moyen de notions empruntées au bouddhisme zen. Selon le moine, il convient à la fois d'acquérir le principe ( $r i$ 理) et le phénomène individuel et concret ( $j i$ 事), c'est-à-dire l'esprit et la technique. Si l'un des deux éléments manque, l'entraînement ne peut être considéré comme suffisant. Le véritable exercice (shugyō修行) ne doit pas négliger ces deux éléments (riji funi 理事不二, union entre principe et phénomène) ${ }^{9}$. Connaître le principe (ri) veut dire parvenir à l'état où l'esprit ne se fixe plus sur un point quelconque - que ce soit au niveau de la posture, de la technique, du sabre de l'adversaire, ou sur quoi que ce soit qui pourrait contrarier la libre circulation de l'esprit. Il faut donc laisser l'esprit se mouvoir dans toutes les directions, sans être figé ni entravé par rien.

8. On suppose que le Taiaki 太阿記 (Traité de l'art ultime du sabre), autre traité qui aborde l'escrime, est également destiné à Munenori, mais ce n'est pas certain.

9. Kamata Shigeo 鎌田茂雄, Kokoro no tatsujin 心の達人 (Le maître de l'esprit), Tōkyō, NHK shuppan NHK出版, 1993, p. 234. 
C'est nourri de cette vision que Munenori plaça dans son Heihō kadensho, pour la première fois de l'Histoire des arts martiaux, la question de l'esprit au centre de l'escrime. Une fois que la technique est bien maîtrisée, ce qui décide finalement de l'issue de l'affrontement, c'est la liberté absolue de l'esprit détaché des gestuelles ${ }^{10}$. Autrement dit, l'entraînement technique est nécessaire pour parvenir à transcender les outils eux-mêmes. C'est en s'entraînant jusqu'à ses dernières limites qu'un sabreur peut se libérer du jeu qu'il exécute et s'affranchir des entraves liées à une technique. Munenori qualifie cet esprit de mushin 無心 (sans esprit) ou bien byōjōshin 平常心 (esprit gardé dans un état d'habitude).

\section{Le nô et l'escrime}

\section{Le lien entre des familles Konparu et Yagyū}

Certes, l'apport de Takuan au Heihō kadensho est considérable, mais il ne faut pas oublier que le contact entre ce moine et Munenori n'a commencé qu'au retour de Takuan à Edo après l'amnistie de juillet 1632, seulement deux mois avant que ce traité ne soit achevé.

On peut raisonnablement considérer que lors de leur rencontre, les textes de Munenori étaient déjà à peu près rédigés. Rappelons en effet que la première partie de ce traité consiste en une transmission de l'enseignement de Hidetsuna, tandis que les deux autres contiennent des réflexions développées par Muneyoshi et Munenori lui-même. On peut supposer qu'à la fin de sa rédaction, Munenori a rajouté des instructions ou des commentaires de Takuan ${ }^{11}$.

Alors, quelle influence le nô a-t-il exercée sur ce traité ? Penchons-nous d'abord sur les relations historiques entre les deux familles Yagyū et Konparu, issues de la même région du Yamato 大和 (Nara aujourd'hui), de lieux distants de seulement deux kilomètres.

10. Minamото Ryōen 源了圓, Kata 型 (La forme), Tōkyō, Sōbunsha 創文社, 1989, p. 208.

11. Nishiyama Matsunosuke, Watanabe Ichirō, Gunji Masakatsu, op. cit., 1972, p. 663. 
Elles se sont rapprochées en particulier à l'ère Keichō 慶長 (1596-1615), à l'occasion d'une rencontre entre Muneyoshi et son élève, Konparu Shichirō Ujikatsu 金春七郎氏勝 ${ }^{12}$ (1575-1610). Préférant dès son adolescence les arts militaires au nô, Ujikatsu apprit non seulement l'escrime, mais aussi la lance, l'équitation, la faux de guerre (naginata 薙刀), et toujours auprès de grands maîtres. Pourtant, il n'était pas le seul acteur à avoir assimilé l'art militaire ; il y en avait d'autres. En effet, grâce à la protection institutionnelle du nô en tant qu'art officiel (shikigaku 式楽) par le gouvernement shogunal au début de l'époque d'Edo, le statut social des acteurs de nô s'était quelque peu rapproché de celui de la classe guerrière. Ce changement a facilité l'accès des acteurs aux arts martiaux $^{13}$.

Passionné surtout par l'escrime, Ujikatsu reçut en 1601 de Muneyoshi le traité Shinkageryū heihō mokuroku no koto 新陰流兵法目録事 (Les explications sur l'art militaire de l'école Shinkage), accompagné d'illustrations qui indiquent les points importants des techniques, ainsi que le recueil Heihō hyakushu 兵法 百首 (Cent poèmes sur l'art militaire). La transmission de cinq autres traités dura jusqu'en 1606. À l'exception du Heihō hyakushu, qui emprunte beaucoup à la pensée confucianiste, tous les autres traités sont caractérisés par des citations d'anciens versets du zen ou l'emploi de termes musicaux ${ }^{14}$.

On peut supposer que le lien qui s'établit entre Muneyoshi et Ujikatsu encouragea le premier à rédiger ses traités, et influença l'esprit dans lequel ils le furent ${ }^{15}$. En effet, Ujikatsu devait avoir bénéficié de la transmission orale de son

12. Le septième successeur à partir de Zenchiku.

13. Imamura Yoshio 今村嘉雄 (dir.), Budō to geidō - Nihon no budō 13 - Soyō (nō, sho, han a, cha）武道と芸道一日本の武道13一素養（能・書・花・茶） (Les arts martiaux et la voie de l'art - Les arts martiaux japonais 13 - La culture [nô, calligraphie, fleur, thé]), Tōkyō, Kōdansha 講談社, 1983, p. 50.

14. Imamura Yoshio 今村嘉雄 (dir.), Nihonbudō taikei dai 1 kan 日本武道大系第1 巻 (Collection d'écrits sur les arts martiaux japonais vol. 1), Kyōto, Dōhōsha shuppan 同 朋社出版, 1982, p. 38.

15. Nishiyama Matsunosuke, Watanabe Ichirō, Gunji Masakatsu, op. cit., 1972, p. 655-657. 
père Anshō 安照 ${ }^{16}$ (Hachirō 八郎, 1549-1621), qui avait reçu en héritage les traités de Zeami, grand créateur et théoricien du nô, et de Konparu Zenchiku.

En échange de l'enseignement que Muneyoshi délivra à Ujikatsu, ce dernier et Anshō enseignèrent le nô à Munenori ${ }^{17}$. Au cours de ces relations, il semble qu' il y ait eu une communication d'arcanes et de secrets entre les familles Konparu et Yagyū : la première a transmis à la seconde une technique pour avancer le pied issoku ikken 一足一見 (un pas, un regard, c'est-à-dire suriashi すり足, pied glissant sur le sol), tandis que la seconde a communiqué à la première la technique désignée par le terme seigōsui 西江水 ${ }^{18}$ (l'eau du fleuve de l'Ouest, c'est-à-dire, exécution du jeu de manière créative sans être enfermé dans la technique ${ }^{19}$ ). À propos de cet échange, Kimura Sukekurō 木村助九郎 (Tomoshige 友 重, 1585-1654), le meilleur élève de Munenori, a noté dans le Heihō kikigaki 兵法 聞書 (Les enseignements notés de l'art militaire, 1632) que Munenori précisait :

Cette affaire d'issoku ikken est conforme à la raison. Elle se retrouve dans la disposition d'esprit du nô de l'école Konparu. C'est vraiment intéressant pour l'art militaire ${ }^{20}$.

D'autre part, Chikamatsu Shigenori 近松茂矩 (1697-1778), vassal du domaine d'Owari 尾張 (ouest d'Aichi), signale dans Mukashi banashi 昔咄 (Vieux récits) :

16. Le sixième successeur à partir de Zenchiku. Il eut la faveur de Toyotomi Hideyoshi ainsi que de Tokugawa Ieyasu.

17. Konparu Anshō 金春安照, Konparu Anshō denshoshū 金春安照伝書集 (Recueil des traités chez Konparu Anshō), Tōkyō, Wan.ya shoten わんや書店, 1978, p. 254.

18. Ce terme provient du Pi-yen-lu. Selon Munenori, « il s'agit de l'instant bref. C'està-dire, très rapide » (Nishiyama Matsunosuke, Watanabe Ichirō, Gunji Masakatsu, op. cit., 1972, p. 660).

19. Shimizu Hiroshi 清水博, Seimeichi toshite no ba no ronri 生命知としての場の論 理 (La logique du champ comme connaissance de la vie), Tōkyō, Chūkō shinsho 中公新 書, 1996, p. 212-213.

20. Nishiyama Matsunosuke, Watanabe Ichirō, Gunji Masakatsu, op. cit., 1972, p. 659. 
Il y a un secret important dit issoku ikken dans l'école Konparu. [...] Pour une certaine raison, la famille Konparu a transmis cette affaire à la famille Yagyū, et, par mesure de réciprocité, cette dernière a communiqué à la famille Konparu l'enseignement secret du seigōsui. C'est pourquoi elles sont devenues les élèves l'une de l'autre, et, de surcroît, un chef de [la confrérie] Konparu a transmis à la famille Yagyū quatre-vingt-treize fameuses pièces écrites de sa main $^{21}$.

Le contact avec le nô par l'intermédiaire de la famille Konparu se reflète dans le Heihō kadensho, par exemple dans ce passage sur la technique du regard 《futatsume tsukai二目遣》(disposition du double regard) ${ }^{22}$ :

Avec diverses tactiques de feinte, on provoque l'adversaire qui se met en attente [tai 待], et alors on regarde comment il réagit. À ce moment-là, on ne le regarde pas en faisant semblant de le regarder, on le regarde en faisant semblant de ne pas le regarder, et, tout en restant vigilant, on jette des regards furtifs vers lui en faisant bouger les yeux, sans fixer ses yeux en un endroit. [...] Il faut agir avec vigilance tout en observant les actions de l'adversaire du coin de l'œil. Dans le nô du sarugaku ${ }^{23}$, il y a ce qu'on appelle la « disposition du double regard»[futame tsukai 二目つかい]. Il s'agit de regarder et aussitôt de détourner le regard. Autrement dit, de ne pas garder les yeux au même endroit ${ }^{24}$.

Dans un autre passage, Munenori signale qu' il faut adopter un rythme différent de celui de l'adversaire, pour le mettre en difficulté quand il voudra frapper, en

21. Ibid., p. 659-660.

22. Ce terme figure dans une note datée de $1710 \mathrm{du}$ journal d'un acteur, Toyooka nikki 豊 岡日記 (Le journal de Toyooka) (Imamura Yoshio [dir.], op. cit., 1983, p. 54).

23. Une ancienne appellation du nô.

24. Nishiyama Matsunosuke, Watanabe Ichirō, Gunji Masakatsu, op. cit., 1972, p. 315 . 
comparant cette situation au rapport entre le chanteur et le joueur de tambour ${ }^{25}$ dans le nô :

Si l'adversaire manie son sabre avec un « rythme lent »[ōbyōshi 大拍子], il faut que nous adoptions un « rythme rapide » [kobyōshi 小拍子]. Si l'adversaire adopte un « rythme rapide », nous devons recourir au « rythme lent ». Ce sont des instructions pour éviter de suivre la mesure de l'adversaire. $S$ 'il parvient à entrer dans votre rythme, il utilisera d'autant plus facilement son arme. Par exemple, quand le chanteur de talent ne le laisse pas entrer dans son rythme, le joueur de tambour médiocre hésite à frapper le tambour. De même qu'il est difficile à un joueur de tambour médiocre d'accompagner un chanteur de talent, ou à un chanteur médiocre de suivre un joueur de tambour de talent, vous ferez en sorte qu'il soit difficile à vos adversaires de chanter ou de frapper ; c'est ce que l'on appelle « à rythme lent rythme rapide, à rythme rapide rythme lent ${ }^{26}$.

Munenori mentionne fréquemment la question du rythme, ce qui montre l'influence du nô ${ }^{27}$. Dans le passage suivant, il compare la relation entre le sabreur et son adversaire à celle entre le danseur ou le chanteur et le musicien : il faut qu'un sabreur connaisse bien la façon dont son adversaire agit, de la même manière qu'un danseur ou un chanteur doit, en apprenant par cœur le shöga章歌, une partition de chant ou de tambour ${ }^{28}$, savoir comment un musicien accompagne la danse ou le chant :

Que ce soit pour la danse ou pour le chant, il ne sera pas possible de bien interpréter sans connaître la partition [shōga]. Dans l'art militaire aussi il faut avoir cet esprit. Fixer les yeux attentivement

25. Il s'agit du tsuzumi 鼓.

26. Nishiyama Matsunosuke, Watanabe Ichirō, Gunji Masakatsu, op. cit., 1972, p. 316.

27. Imamura Yoshio (dir.), op. cit., 1983, p. 55.

28. Ibid., p. 55. 
sur les mouvements ou la manipulation du sabre chez l'adversaire, et pénétrer jusqu'aux tréfonds de ses intentions : cela doit être l'esprit dans lequel on apprend par cœur la partition [shöga] pour la danse et le chant. La connaissance intime des mouvements et du style de votre adversaire vous donne une plus grande liberté d'action ${ }^{29}$.

La remarque sur la partition (shōga) figure également dans Tsuki no shō 月 之抄 (Les notes de la lune, 1642), écrit par Yagyū Jūbei Mitsuyoshi 十兵衛三 撖 (1607-1650), le fils de Munenori :

On dit aussi que l'exécution d'une partition [shōga] est une affaire de souffle. Il faut savoir comment accorder son souffle avec l'autre. Lorsque l'adversaire est en « rythme rapide », et qu'il est difficile de suivre son rythme, on sera soulagé si l'on retient le souffle en émettant un cri « Yah ! ». Pousser le cri permet de s'accorder avec le rythme requis et de se laisser porter par le rythme. [...] Il en va de même pour le rythme du momidashi $i$ 揉出 ${ }^{30}$.

Dans cette technique appelée momidashi, une frappe du grand tambour intervient par intervalles entre les frappes du petit tambour ${ }^{31}$ qui marquent le rythme avec constance ${ }^{32}$. En d'autres termes, la disposition du souffle du sabreur par rapport à son adversaire qui adopte un rythme rapide est comparée à celle du grand tambour par rapport au petit tambour.

29. Nishiyama Matsunosuke, Watanabe Ichirō, Gunji Masakatsu, op. cit., 1972, p. 316.

30. Imamura Yoshio (dir.), op. cit., 1983, p. 164. Momidashi est une technique du grand tambour (ózutsumi 大鼓) dans la séquence appelée momi no dan 揉ノ段 de la pièce de nô Okina翁 où se déploie une danse dynamique (躍動的な舞).

31. Il s'agit du kozutsumi 小鼓.

32. Koyama Hiroshi 小山弘志 (dir.), Iwanami kōza nō kyōgen 岩波講座・能・狂言 (Cours Iwanami, Nô Kyōgen) VI Nō kanshō annai 能鑑賞案内 (Introduction à la mise en scène du nô), Tōkyō, Iwanami shoten 岩波書店, 1989, p. 16. 
Dans un autre passage, Mitsuyoshi compare l'escrime au nô, en citant Konparu Yasuyoshi 金春安喜 (Hachizaemon 八左衛門, 1588-1661), le fils cadet d'Anshō :

Dans la disposition d'esprit du nô de l'école Konparu, il y a un souci du rythme qui se détermine en fonction de l'autre, non pas de soi. Il s'agit d'apaiser son ego et d'exécuter [le jeu] selon le niveau de l'autre. Le vieux Hachirōzaemon l'a raconté. Comme c'est intéressant, je le note ${ }^{33}$.

De même, selon Mitsuyoshi, pour connaître son adversaire au sabre, il faut modérer son ego au point d'être capable de l'oublier, et adapter ses dispositions en fonction de son adversaire. Car la volonté de battre un adversaire empêche de l'observer, entraînant des mouvements inadéquats.

\section{Konparu Anshō et son héritage depuis l'époque de Zeami}

L'influence du nô sur le Heihō kadensho paraît évidente. On avance même l'hypothèse d'une influence directe de Konparu Anshō sur ce traité ${ }^{34}$.

Quelle était la vision de cet acteur ? Inspiré par des idées confucianistes et bouddhiques, il a laissé les traités intitulés Konparu Anshō hidensho 金春 安照秘伝書 (Livre de la transmission secrète de Konparu Anshō, 1606) et Konparu Anshō nōdensho 金春安照能伝書 (Livre de la transmission du nô de Konparu Anshō, 1610), en suivant les traces de Zenchiku ou de son petit-fils Zenpō 禅鳳 35 (Hachirō Motoyasu 八郎元安, 1454-vers 1532). Insatisfait du nô de son époque, surtout préoccupé de l'aspect technique, Anshō a insisté sur la disposition de l'esprit qui permet de se détacher des désirs, par exemple celui de susciter chez le spectateur un cri d'admiration ${ }^{36}$.

33. Imamura Yoshio (dir.), op. cit., 1983, p. 204.

34. Konparu Anshō, op. cit., 1978, p. 255.

35. Le troisième successeur à partir de Zenchiku.

36. Konparu Anshō, op. cit., 1978, p. 245. 
Comme l'avait fait Takuan, cet acteur compare l'esprit au principe $r i$ 理 et le corps au phénomène ( $j i$ 事) ${ }^{37}$, en signalant que « l'esprit et le corps sont comme les deux roues d'une voiture ${ }^{38}$. Le ri est dit également « esprit foncier » (honshin 本心) ou 《esprit sans intervention de la moindre pensée » (mushin munen 無 心無念 $)^{39}$. En somme, à l'instar de Takuan ou de Munenori, Anshō a mis l'accent sur le fait que l'attention ne doit pas être arrêtée par le geste à exécuter :

$\mathrm{Si}$, dès le noviciat, l'on se consacre exclusivement à s'approprier des gestuelles, on exécute spontanément le jeu sans penser aux choses, sans savoir, sans artifice, et l'esprit ne s'arrête sur aucun geste. De ce fait, le geste disparaît naturellement et seule la véritable intention du nô émerge ${ }^{40}$.

Lorsque l'intention d'effectuer une chose surgit, l'esprit y demeure et l'articulation entre les gestes sera entravée et bloquée ${ }^{41}$.

Il est possible d'expliquer cette proximité entre Anshō et Munenori par le zen, dont tous deux étaient familiers. Mais on peut supposer qu'elle provient de l'influence directe d'Anshō sur Munenori : il est possible que le contenu des traités

37. Cette remarque reflète une pensée répandue à l'époque, car ces concepts figurent également dans le Nanbōroku 南方録 (Les enseignements notés par Nanbō, vers 1650) qui note les enseignements de Sen no Rikyū 千利休 (1522-1591), maître du thé : «En fait, le phénomène et le principe ne sont pas séparés. Si le phénomène est mûr, l'esprit le devient, et si l'esprit est mûr, le métier le devient. Si, bien qu'on exécute convenablement le métier, l'esprit n'atteint pas encore l'aboutissement, c'est parce que le métier n'y est pas encore parvenu non plus. Si, bien que l'esprit atteigne la maturité, le métier ne parvient pas au niveau ultime, c'est parce que l'esprit n'a pas encore pénétré jusqu'au fond » (Nishiyama Matsunosuke, Watanabe Ichirō, Gunji Masakatsu, op. cit., 1972, p. 96).

38. Dans le bouddhisme, l'exercice (shugyō) portant sur le $r i$ 理et le $j i$ 事s'appelle shinshin gakudō 身心学道 (apprendre par le corps et par l'esprit) (Kamata Shigeo, op. cit., 1993, p. 285).

39. Konparu Anshō, op. cit., 1978, p. 147, 167.

40. Ibid., p. 169.

41. Ibid., p. 175. 
de cet acteur ait été transmis oralement à Munenori ou à Ujikatsu, même si ces traités n’ont été transmis sous forme écrite qu’à Nakamura Katsusaburō 中村勝 三郎 (1572?-1644), acteur de nô servant auprès du daimyo à Kumamoto ${ }^{42}$.

Ici, il faut signaler que la vision d'Anshō s'est nourrie de transmissions orales ou écrites remontant notamment à trois de ses aïeux : Zenpō, son arrière-grandpère, Zenchiku, grand-père de Zenpō, et Zeami, dont Zenchiku était le gendre.

Zeami a dédié à son héritier effectif Zenchiku ${ }^{43}$ les traités Rikugi 六義 (Les six modes, 1428), Shügyoku tokka 拾玉得花 (Amasser les joyaux, atteindre la fleur, 1428). Il semble par ailleurs que ce dernier a eu l'occasion de transcrire les autres traités appartenant à Motomasa 元雅 (1400?-1432), fils aîné de Zeami, tels que Füshikaden 風姿花伝 (De la transmission de la fleur de l'interprétation, 1400), Kakyō 花鏡 (Le miroir de la fleur, 1424), Shikadō 至花道 (La voie qui mène à la fleur, 1420), Goi 五位 (Les cinq degrés, non daté), Nikyoku santai ningyōzu 二曲三体人形図 (Étude des figures humaines des deux éléments et des trois types, 1421), Kyūi 九位 (Les neuf degrés, non daté). Selon les documents de la famille Konparu, tous ces traités, à l'exception de Kyüi et de certains textes de Zenchiku ou Zenpō, ont été transmis à la génération d'Anshō ${ }^{44}$.

Au vu de la proximité entre Zeami et Zenchiku, et entre ce dernier et Zenpō, on peut imaginer que les idées de Zeami ont également pu être transmises oralement jusqu'à la génération de Zenpō, qui les a ensuite léguées à ses descendants tels Anshō ou Ujikatsu.

42. Ibid., p. 255.

43. En raison de la mort précoce de son fils Motomasa, ce fut son neveu Motoshige 元 重 (Onnami 音阿弥 1398-1467) qui succéda à Zeami en devenant le troisième chef de la compagnie Kanze. Néanmoins, ce dernier n'a reçu directement aucun traité de Zeami, ni laissé aucun traité lui-même. Zenchiku est considéré par Zeami comme faisant partie de la famille et comme son unique successeur possible après la mort de Motomasa (ZEAMI 世 阿弥 et ZENCHIKU 禅竹, compilé par OMOTE Akira 表章 et KATō Shūichi 加藤周一, Zeami Zenchiku 世阿弥・禅竹 [Zeami et Zenchiku], Tōkyō, Iwanami shoten 岩波書 店, 1974, p. 574).

44. Омоте Akira 表章 et ITŌ Masayoshi 伊藤正義, Konparu kodensho shūsei 金春古 伝書集成 (Recueil des anciens traités de Konparu), Tōkyō, Wan.ya shoten わんや書 店, 1969, p. 20-22, 29. 
Il faut signaler que des affinités existaient déjà entre les écrits de Zeami et les traités d'art militaire. À l'époque de Zeami avaient lieu des concours de nô, dits tachiai立合, qui permettaient de comparer les mérites de différentes confréries qui jouaient chacune à leur tour une pièce différente. La réussite à ces concours assurait la protection des autorités et était pour les compagnies un enjeu vital dans un contexte de concurrence acharnée. Dans Füshikaden, Zeami analyse les stratégies pour l'emporter en comparant le concours de nô à une guerre. Ses traités décrivent des stratégies concrètes pour survivre en tant qu'acteur et l'emporter sur les autres, considérés comme des adversaires, en présentant un spectacle excellent ${ }^{45}$.

La réflexion sur le théâtre de Zeami est influencée par le zen, notamment dans la dernière période de sa vie. Son lien avec l'école Sōtō 曹洞宗du zen est souligné par Kōsai Tsutomu 香西精 : il rappelle que Zeami prit l'habit au Fuganji 補螈 寺, temple de la secte Sōtō, en 1422, et relève plusieurs termes chez cet acteur qui démontrent l'influence de cette école ${ }^{46}$. Cependant, la plupart des chercheurs considèrent que Zeami n'a été influencé par le zen que de manière passive ${ }^{47}$, dans la mesure où dans ses écrits les citations du bouddhisme ne servent que de cadres de comparaisons, et qu'il serait possible d'exposer les mêmes idées en recourant à d'autres métaphores. À mon sens également, le bouddhisme, surtout le zen, n'est pour Zeami qu'un moyen ou un outil conceptuel pour comprendre, analyser et exprimer ce qu'il a découvert à travers ses expériences effectives sur scène ${ }^{48}$.

Certes, Munenori emprunte aussi des termes au zen pour expliquer la pratique du sabre, mais lui a noué un lien particulier avec Takuan, lequel a analysé la pratique du sabre en s'appuyant sur le zen. Autrement dit, chez Munenori, il y a un contact direct entre l'escrime et le zen.

Quel que soit le lien avec le zen, l'aboutissement ultime est, chez Zeami et Munenori, le détachement de toute attention envers le jeu. Munenori illustre ce

45. ZEAMI et ZENCHIKU, op. cit., 1974, p. 30, 519-521.

46. Kumakura Isao 熊倉功夫 (dir.), Sōsho Zen to nihonbunka 3 : Zen to nōgaku, cha, 趣書禅と日本文化3一禅と能楽・茶 (Collection Le zen et la culture japonaise 3 - Le zen et le nô, la cérémonie du thé), Tōkyō, Perikansha ペりかん社, 1997, p. 187-242.

47. Ibid., p. 374, 375.

48. ZeAmi et ZenCHIKU, op. cit., 1974, p. 519. 
principe au moyen de la métaphore suivante : « La pierre parfaitement polie ne sera pas souillée même dans la fange ${ }^{49}$. La même idée est exprimée par Zeami de manière analogue :

Il [le maitre zen Zide Huihui] dit également que « l'or pur ne change pas de qualité même dans les flammes, et la pierre blanche garde sa figure parfaite même dans la fange $\gg^{50}$. Il en est ainsi de notre art. Une fois qu'il a assimilé les différents degrés de la fleur [hana] - depuis les trois moyens jusqu'aux trois supérieurs -, même quand il interprète des pièces relevant des trois degré [kurai] inférieurs, l'acteur doit rester au niveau établi et stable des trois degrés supérieurs de la fleur. C'est-à-dire que, même quand l'or se mélange au sable ou que la fleur de lotus est plongée dans la fange, ils n'en sont point affectés. Le maître confirmé qui est parvenu à ce niveau ne doit se situer qu'au véritable degré de l'aisance ${ }^{51}$. Quelque registre qu'il interprète, il ne doit pas penser « c'est facile » dans son esprit. Il s'agit d'un jeu interprété sans aucun artifice ni conscience [mukyoku mushin no 無曲・無心の]. Ce degré doit être appelé la fleur merveilleuse qui atteint le véritable néant ${ }^{52}$.

Un autre exemple, l'expression « oiseau marin » dans Sarugaku dangi 申楽談 儀(Entretiens sur le sarugaku, 1430) de Zeami, nous permet de suggérer aussi la transmission au fil des générations depuis cet acteur jusqu’à Munenori :

49. Nishiyama Matsunosuke, Watanabe Ichirō et Gunji Masakatsu, op. cit., 1972, p. 320.

50. Il s'agit du sixième verset de Roku gyü-zu 六牛図 (Six dessins du buffle) de Zide Huihui 自得慧暉 (en japonais Jitoku Keiki, 1097-1183), maître zen de l'école Caotong (en japonais Sōtō) 曹洞.

51. Le degré d'aisance (an.i 安位ou yasuki kurai 安き位) veut dire un niveau où l'on devient maître de son propre art après des exercices soutenus, lorsque même des jeux extrêmement difficiles sont exécutés avec la plus grande facilité (ZEAMI et ZENCHIKU, op. cit., 1974, p. 92).

52. Ibid., p. 189, 190. 
[Il y a celui qui] imite sans réussir [les grands acteurs] qui chantent joliment en apparence, en ayant le rythme, mais avec des efforts importants et sans être perçu comme un oiseau marin ${ }^{53}$.

Cette comparaison figure aussi dans Söin sodeshita 宗筠袖下 (Le secret de Sōin), un traité apocryphe de Konparu Shichirō Motouji 金春七郎元氏 (Sōin 宗筇, 1432-1480), fils de Zenchiku :

Le chant est comme un oiseau marin. L'apparence paraît tranquille, mais si un oiseau marin relâche la vigilance portée à l'esprit, à ses pattes, il s'enfonce dans l'eau ${ }^{54}$.

Zenpō, le fils de Sōin, reprenant le discours de Zeami ${ }^{55}$, note dans son Mōtan shichinshō 毛端私珍抄 (Notes de certains enseignements transmis en secret) :

Il faut prendre soin de ne rien dévoiler, à l'instar de la rivière Yodogawa qui, calme en surface, coule rapidement en profondeur. Il s'agit de la comparaison avec l'oiseau marin (qui figure dans le Sarugaku dangi) ${ }^{56}$.

L'expression « oiseau marin » figure également dans le Heihō kadensho :

Dans l'art militaire aussi, votre énergie doit rester active à l'intérieur; en gardant la vigilance, on offre une apparence tranquille à l'extérieur sans s'agiter. [...] Comme l'oiseau marin flottant sur

\section{Ibid., p. 282.}

54. Омоте Akira et Iто̄ Masayoshi, op. cit., 1969, p. 530.

55. Омоте Akira 表章, ТАкемото Mikio 竹本幹夫, Iwanami kōza Nō kyōgen 岩波 講座能 • 狂言 (Cours Iwanami, Nô et Kyōgen) II, Nōgaku no densho to geiron 能楽の 伝書と芸論 (Les traités et la théorie artistique du nô), Tōkyō, Iwanami shoten 岩波書 店, 1988, p. 250 .

56. Омоте Akira, Iто̄ Masayoshi, op. cit., 1969, p. 338. 
l'eau agite ses pattes palmées sous l'eau et paraît tranquille en surface, il faut garder la vigilance à l'intérieur... ${ }^{57}$

Il est clair que cette métaphore, présente dans les traités de nô et dans ceux de Munenori, a toujours la même signification.

En somme, il est fort probable qu'ayant une connaissance des idées de Zeami grâce à sa relation avec Anshō et son fils Ujikatsu, Munenori les a introduites dans son Heihō kadensho. Même si ce n'est pas de manière directe, on peut considérer que les traités de Zeami ont exercé une certaine influence sur le Heihō kadensho ${ }^{58}$. Autrement dit, Munenori a absorbé la pensée zen non seulement à travers son contact avec Takuan, mais aussi à partir de la vision de Zeami, lequel avait déjà utilisé et assimilé cette pensée pour le nô.

\section{Le nô et la classe guerrière}

Il est certain que les échanges entre les familles Konparu et Yagyū étaient importants, mais le nô et l'escrime étaient déjà entrés en contact avant même ce rapprochement car le nô avait, dès sa naissance, entretenu un lien étroit avec la haute classe guerrière. Ayant connu un essor et un développement considérable grâce au soutien personnel accordé par le shōgun Ashikaga Yoshimitsu 足利義 満 (1358-1408) à Kan.ami 観阿弥 (Kiyotsugu 清次, 1333-1384) et à son fils Zeami, le nô a toujours bénéficié de la protection du gouvernement shogunal. Avant l'ère Ōnin 応仁 (1467-1469), il avait déjà acquis le statut d'art que les guerriers se doivent d'apprécier ${ }^{59}$.

Pourtant, les troubles de l'ère Ōnin (応仁の乱 Ōnin no ran, 1467-1477) à Kyoto compromirent la situation financière du gouvernement shogunal qui

57. Nishiyama Matsunosuke, Watanabe Ichirō, Gunji Masakatsu, op. cit., 1972, p. 319 .

58. Imamura Yoshio (dir.), op. cit., 1983, p. 57, 63.

59. Омоте Akira, Amano Fumio 天野文雄, Iwanami kōza Nō kyōgen 岩波講座 能・ 狂言 (Cours Iwanami, Nô et Kyōgen) I, Nōgaku no rekishi能楽の歴史 (Histoire du nô), Tōkyō, Iwanami shoten 岩波書店, 1987, p. 69. 


\section{CIPANGO}

248 Cahiers d'études japonaises n²2

assurait le patronage du nô. Les acteurs devaient désormais trouver d'autres revenus, notamment en enseignant le chant (utai 謡) ou la danse du nô (shimai 仕舞). Ainsi, des guerriers se mirent à pratiquer la musique ou la danse du nô ${ }^{60}$.

À la fin de l'époque de Muromachi, divers arts d'agrément étaient pratiqués dans la haute société guerrière, ce qui a permis un rapprochement entre les arts d'agrément et les arts militaires qui partageaient souvent les mêmes conceptions esthétiques $^{61}$. Cette situation culturelle se reflète dans les écrits de Zenpō, où il est question d'arts comme l'escrime, l'équitation, la flûte shakuhachi 尺八, le luth biwa 琵琶, la cithare koto 琴, l'arrangement floral, la balle (mari 鞠), le thé, la poésie, etc ${ }^{62}$.

Ainsi, dans le Zenpō zōdan 禅鳳雑談 (Causerie sur l'art de Zenpō, 1513), Zenpō compare le sabre à l'éventail :

Il faut que vous teniez un éventail comme on porte un sabre dans l'école orthodoxe [tōryū 当流] ${ }^{63}$ de l'art militaire. Si vous imaginez porter un sabre ou un petit sabre, il n'arrivera pas que vous laissiez tomber un éventail ${ }^{64}$.

On ne peut identifier l'« école orthodoxe de l'art militaire » dont il est question ici, mais on peut supposer qu'à l'ère Eishō永正 (1504-1520), quand ce traité a été rédigé, existaient déjà les bases d'une relation entre la famille Konparu

60. Hayashiya Tatsusaburō 林屋辰三郎, Kodai chūsei geijutsuron 古代中世芸術論 (Théorie artistique antique et médiévale), Tōkyō, Iwanami shoten 岩波書店, 1973, p. 798.

61. Nakamura Yasuo 中村保雄, 《Shin gyō sōno sekai » 真行草の世界 (Le monde des formel, semi-formel, informel), in NaKAMURA Yasuo (dir.), Nihon geinōshi - dai 3 kan $c h \bar{u} s e i$ 日本芸能史 - 第 3 巻 - 中世 (Histoire d'art japonais, vol. III, Moyen Âge), Tōkyō, Hōsei daigaku shuppankyoku 法政大学出版局, 1983, p. 344.

62. Hayashiya Tatsusaburō, op. cit., 1973, p. 796-797.

63. Ici, ce terme doit être entendu d'une école dont la manière est correcte, par opposition aux « écoles secondaires » (taryū 他流) dont la manière est singulière et moins efficace (IMAMuRa Yoshio [dir.], op. cit., 1983, p. 38-39).

64. Hayashiya Tatsusaburō, op. cit., 1973, p. 486. 
et le milieu de l'escrime, et que ces relations se sont développées ultérieurement avec la famille Yagyū vers l'ère Keichō ${ }^{65}$.

Dans un autre traité, le Hogoura no sho 反古裏の書 ${ }^{66}$ (Écrit rédigé au revers de vieux papiers), Zenpō compare également le nô à l'art militaire à propos de l'éventail :

La posture adoptée lorsqu'on porte un éventail illustre l'esprit de l'art militaire de l'école orthodoxe ${ }^{67}$.

Dans le Zenpō zōdan, il évoque encore l'art militaire dans une remarque sur la manière de battre le rythme avec un éventail :

Il faut savoir que les différentes manières de faire caractéristiques - comme tourner ou tordre un éventail - se retrouvent dans des écoles secondaires [tary $\bar{u}$ 他流] de l'art militaire. Dans tous les cas, il est bon de frapper à la manière de l'école orthodoxe ${ }^{68}$.

Enfin, dans ce même traité, il affirme clairement l'existence d'un rapport entre le nô et l'escrime : « L'art militaire et le jeu de la balle au pied (kemari 蹴鞠) sont proches du nô ${ }^{69}$.

65. Ibid., p. 507.

66. Le terme hogo désigne une feuille de papier gâchée par une calligraphie manquée.

67. Омоте Akira, Iтō Masayoshi, op. cit., 1969, p. 356.

68. Hayashiya Tatsusaburō, op. cit., 1973, p. 488.

69. Ibid., p. 502. 
D'autre part, la communication entre ces deux pratiques se retrouve aussi dans la posture fondamentale du nô appelée kamae 構 $亢^{70}$, dont on pense qu'elle a été importée de l'escrime à cette époque.

L'origine de cette posture pourrait remonter au traité du jeu de la balle au pied (kemari), le Kemari kudenshu $\bar{u}$ 蹴鞠口伝集 (Recueil de transmission orale du jeu de la balle au pied, vers 1150), dans lequel l'importance de baisser les reins est signalée. Ce point n'est pas mentionné par Zeami mais on sait, grâce au témoignage de Nijō Yoshimoto 二条良基 (1320-1388), poète, intellectuel et grand chancelier, que cet acteur de nô était habile au jeu du kemari («il est doué même pour le renga [poèmes en chaîne] et le ballon », dit-il de Zeami). Il est donc possible que Zeami ait connu cette posture de base ${ }^{71}$.

Dans le Zenpō zōdan, Zenpō affirme qu'il faut « avoir la force dans le tronc» pour la danse, faute de quoi « ce sont les mains qui dansent », ce qui n'est pas souhaitable ${ }^{72}$. Il semble que c'est vers la fin du $\mathrm{XVI}^{\mathrm{e}}$ siècle que le terme kamae dans son sens actuel apparut : dans le Hachijō kadensho 八帖花伝書 (Livre

70. Cette posture, dont proviennent tous les mouvements du nô, s'obtient en abaissant le centre de gravité du corps. Ce n'est pas une simple position statique, mais une position qui provient d'un équilibre entre les forces tirant le corps vers l'avant et l'arrière, vers le haut et vers le bas (Yокомісні Mario 横道萬里雄, Iwanami köza Nō kyōgen 岩波講座 能 - 狂言 [Cours Iwanami, Nô et Kyōgen] IV, Tōkyō, Iwanami shoten 岩波書店, 1987, p. 269). Cette posture comporte en même temps un aspect psychique, ce dont témoigne l'expression kokoro gamae 心構え (littéralement, « disposition, posture de l'esprit », qui signifie : « préparation mentale », « attention »), où l'on retrouve le même terme kamae (posture).

71. À ce propos, Ishii Tomoko a effectué des recherches sur le rapport entre le kemari (jeu de la balle au pied), le nô, et l'art militaire (IsHII Tomoko 石井倫子, Furyūnō no jidai - Konparu Zenpō to sono shühen 風流能の時代一金春禅鳳とその周辺 [L'époque du nô du furyū - Konparu Zenpō et son entourage], Tōkyō, Tōkyō daigaku shuppankai 東 京大学出版会, 1998).

72. HaYAShiYa Tatsusaburō, op. cit., 1973, p. 490. 
de transmission de la fleur en huit fascicules) ${ }^{73}$, avec le dessin d'un homme qui illustre cette posture, il est noté qu' il faut une « stabilisation au niveau du tronc » (dozzukuri 胴づくり), sans quoi « les reins flottent $»^{74}$. Matsuoka Shinpei 松岡 心平 commente ainsi la relation entre le nô et les arts guerriers :

L'art militaire et le nô exigent cette même forme de concentration caractéristique du zen. Peut-on dès lors considérer qu'à travers une communication effective entre un maître du sabre et un acteur de nô, la position du corps de l'art militaire s'est transmise au nô, et qu'ainsi s'est formé petit à petit le kamae (qui permet) la «stabilisation au niveau du tronc »? Le terme kamae peut aussi provenir d'autres expressions utilisées dans l'art militaire, telles que le seigan no kamae 正眼の構え (posture dans laquelle, en garde, on oriente la lame vers les yeux de l'adversaire) ${ }^{75}$.

Plus que tout autre, Toyotomi Hideyoshi a rapproché le nô de la classe guerrière et, à l'instar d'Ashikaga Yoshimitsu, fut un grand protecteur de cet art. Ayant commencé à apprendre lui-même le nô en 1592, Hideyoshi en devint rapidement un acteur passionné. Cet enthousiasme se manifesta dès l'année suivante, lorsqu'il organisa trois jours de spectacle nô dans son palais, au cours desquels il interpréta lui-même douze pièces, accompagné de généraux tels que Tokugawa Ieyasu et d'acteurs professionnels comme Konparu Anshō, favori de Hideyoshi. Non seulement il était lui-même un défenseur enthousiaste du nô, mais il aurait aussi vivement recommandé à son entourage de pratiquer cet art. Sa

73. Traité compilé à partir d'une partie du Füshikaden et de l'Ongyoku kuden 音曲口 伝 (Transmission orale du chant, 1419) de Zeami, ainsi que d'autres traités secrets, probablement dans la dernière moitié de l'ère Tenshō 天正 (1573-1592). Éditeur inconnu.

74. Hayashiya Tatsusaburō, op. cit., 1973, p. 597 ; Matsuoka Shinpei 松岡心平 (dir.), Zeami chüsei no geijutsu to bunka 01 : Zeami to sono jidai 世阿弥 中世の芸術と文化 世阿弥とその時代 (Zeami, L'art et la culture au Moyen Âge, Zeami et son époque), Tōkyō, Shinwasha 森話社, 2002, p. 177-182.

75. IsHir Tomoko, op. cit., 1998, p. 182. 
252 Cahiers d'études japonaises n²2

passion et le soutien qu'il accorda aux quatre confréries du Yamato ont contribué à l'émergence d'une période florissante du nô ${ }^{76}$.

La dévotion pour le nô de Hideyoshi peut paraître insolite, mais, à l'ère Tenshō (1573-1592), de nombreux guerriers appréciaient et soutenaient le nô, même dans les provinces. Des groupes chantant du nô, les utai-kō 謡講 se multiplièrent dans la société guerrière ${ }^{77}$.

À l'époque d'Edo, la culture indispensable pour la classe guerrière imposait d'avoir quelques connaissances sur le nô ou le chant ${ }^{78}$. Parmi les samouraïs de cette période, Munenori fut un des plus grands amateurs de nô. Son excès de passion - il dansait chez le daimyo où il s'était invité - est même critiqué par Takuan à la fin du traité Fudōchi shinmyōroku. Selon ce document, depuis 1635, date où Munenori a interprété la pièce Takasago 高砂 au château d'Edo, il a joué un total de quarantesix fois (vingt-trois pièces de nô) lors de cérémonies organisées par Iemitsu. Il semble qu'il se soit encore davantage consacré à cet art dans la dernière période de sa vie, sans doute parce qu'à cette époque Iemitsu appréciait les représentations de nô jouées par des amateurs. On peut supposer que Munenori avait commencé à pratiquer le nô plus tôt.

Le plus remarquable, c'est que Munenori interpréta à trois reprises, en 1637, 1639, et 1641, Sekidera Komachi 関寺小町, une pièce considérée comme «secrète »( hikyoku 秘曲 $\left.{ }^{79}\right)$, la plus prestigieuse de celles dont le protagoniste est une vieille femme (rōjō-mono 老女物 $\left.{ }^{80}\right)$. Cette pièce a une telle importance que, lors de sa présentation en 1634 par Kita Shichidayū 喜多七大 夫 (1586-1653), en raison de sa mise en scène distinguée et des anciens usages, cet acteur ainsi que les trois musiciens qui l'accompagnaient avaient été confinés à

76. Омоте Akira, Amano Fumio, op. cit., 1987, p. 81-86.

77. Ibid., p. 86.

78. Imamura Yoshio (dir.), op. cit., 1983, p. 51.

79. Une pièce transmise exclusivement aux acteurs ayant un lien familial avec le chef d'une école ou reconnus comme étant doués d'un talent exceptionnel.

80. Ce répertoire, qui exige une grande habileté, un haut degré artistique, ainsi qu'une excellente maîtrise mentale, est réservé aux acteurs ayant atteint un certain âge (Koyama Hiroshi [dir.], op. cit., 1989, p. 125). 
leur domicile par les autorités sans autorisation de sortir ${ }^{81}$. Le fait que Munenori se soit permis d'interpréter cette même pièce à peine quelques années après cette mesure extrêmement stricte montre qu'il avait reçu une autorisation officielle pour l'exécuter, autrement dit qu'elle lui avait été enseignée par transmission directe d'un acteur ayant rang de chef de confrérie - probablement Ujikatsu ou son père Anshōo ${ }^{82}$.

\section{Conclusion}

Sa relation intime avec la famille Konparu permit donc à Munenori de connaître les enseignements secrets du nô et, en même temps, de pratiquer cet art. Ainsi, il a été inspiré par cette forme artistique au plan à la fois conceptuel et pratique.

Certes, l'apport du zen, notamment de Takuan, a été considérable en ce qui concerne la maîtrise mentale nécessaire pour l'escrime, mais la disposition d'esprit a été soulignée également par un acteur de nô tel que Konparu Anshō, qui avait baigné dans la transmission orale et écrite issue de plusieurs générations de maîtres de nô depuis Zeami. Autrement dit, le sabre a été inspiré par le nô, qui avait déjà incorporé et digéré le zen, tant du point de vue conceptuel que de celui de l'esthétique de la gestuelle, environ deux siècles avant que l'escrime n'intègre cette pensée. On peut dire qu'au moment où l'art guerrier se constitua en tant que voie, le nô joua un rôle de référence importante.

L'influence du nô sur le sabre n'est pourtant pas intervenue du jour au lendemain, à l'occasion du rapprochement entre les deux familles Yagyū et Konparu. Cette base avait été préparée depuis plus d'un siècle par la relation profonde et étroite qui s'était formée entre la classe guerrière et cette forme théâtrale.

Enfin, l'escrime et le nô partagent une affinité en termes de pratique, par les techniques fondamentales telles que le pas, la posture ainsi que le rythme qui permettent d'établir une relation entre le sabreur et son adversaire - comme entre artistes sur scène.

81. Imamura Yoshio (dir.), op. cit., 1983, p. 53.

82. Ibid., p. 18, 51-53. 
N.B. : Pour la traduction française des textes de Munenori, je me suis référée aux ouvrages suivants, m'autorisant à modifier les traductions quand cela m'a paru nécessaire :

Yagyū Munenori, trad. Nickels-Grolier Josette, Le Sabre de vie - Les enseignements secrets de la maison du Shôgun, Noisy-sur-École, Budo Éditions, 2005.

Yagyū Munenori, TAkuan, trad. Cleary Thomas, L'Âme du samouraï : Une traduction contemporaine de trois classiques du Zen et du Bushidô, Monaco, Éditions du Rocher, 2007.

\section{Bibliographie}

HaYAshiYa Tatsusaburō 林屋辰三郎, Kodai chūsei geijutsuron 古代中世芸術 論 (Théorie artistique antique et médiévale), Tōkyō, Iwanami shoten 岩波書 店, 1973.

ImAmuRa Yoshio 今村嘉雄 (dir.), Nihonbudō taikei dai 1 kan 日本武道大系第 1 巻 (Collection d'écrits sur les arts martiaux japonais vol. 1), Kyōto, Dōhōsha shuppan 同朋社出版, 1982.

ImAmuRa Yoshio 今村嘉雄 (dir.), Budō to geidō-Nihon no budō $13-$ Soyō (nō, sho, hana, cha）武道と芸道一日本の武道13一素養（能・書・花 茶) (Les arts martiaux et la voie de l'art - Les arts martiaux japonais 13 - La culture [nô, calligraphie, fleur, thé]), Tōkyō, Kōdansha 講談社, 1983.

Ishiguro Kichijirō 石黒吉次郎, Chūsei geidōron no shisō - Kenkō, Zeami, Shinkei 中世芸道論の思想一兼好 - 世阿弥 - 心敬 (La pensée de la conception artistique au Moyen Âge - Kenkō, Zeami, Shinkei), Tōkyō, Kokusho kankōkai 国書刊行会, 1993. 
IsHII Tomoko 石井倫子, Furyūnō no jidai - Konparu Zenpō to sono shūhen 風流能の時代一金春禅鳳とその周辺 (L'époque $\mathrm{du}$ nô $\mathrm{du}$ fury $\bar{u}$ - Konparu Zenpō et son entourage), Tōkyō, Tōkyō daigaku shuppankai 東 京大学出版会, 1998.

Kamata Shigeo 鎌田茂雄, Kokoro no tatsujin 心の達人 (Le maître de l'esprit), Tōkyō, NHK shuppan NHK出版, 1993.

KonisHi Jin.ichi 小西甚一, Michi - chüsei no rinen 道一中世の理念 (La voie - Un concept du Moyen Âge), Tōkyō, Kōdansha 講談社, 1975.

Konparu Anshō 金春安照, Konparu Anshō denshoshū 金春安照伝書集 (Recueil des traités chez Konparu Anshō), Tōkyō, Wan.ya shoten わんや書 店, 1978 .

Koyama Hiroshi 小山弘志 (dir.), Iwanami kōza Nō kyōgen 岩波講座・ 能・狂言 (Cours d'Iwanami Nô Kyōgen) VI Nō kanshō annai 能鑑賞案 内 (Intrduction à la mise en scène du nô), Tōkyō, Iwanami shoten 岩波書 店, 1989 .

Kumakura Isao 熊倉功夫 (dir.), Sōsho Zen to nihonbunka 3 : Zen to nōgaku, $c h a$, 叢書禅と日本文化3一禅と能楽・茶 (Collection Le zen et la culture japonaise 3 - Le zen et le nô, la cérémonie du thé), Tōkyō, Perikansha ペり かん社, 1997.

KURASAWA Yukihiro 倉沢行洋, Geidō no tetsugaku - shūkyō to geino sōsoku 芸 道の哲学一宗教と芸の相即 (La philosophie de la voie de l'art - La fusion entre la religion et l'art), Ōsaka, Tōhō shuppan 東方出版, 1983.

Matsuoka Shinpei 松岡心平 (dir.), Zeami chüsei no geijutsu to bunka 01 : Zeami to sono jidai 世阿弥・中世の芸術と文化 01 世阿弥とその時代 (Zeami, L'art et la culture au Moyen Âge, 01 : Zeami et son époque), Tōkyō, Shinwasha 森話社, 2002. 


\section{CIPANGO}

256 Cahiers d'études japonaises n²2

Minamoтo Ryōen 源了圓, Kata 型 (La forme), Tōkyō, Sōbunsha 創文社, 1989.

NaKamura Yasuo 中村保雄, 《Shin gyō sō no sekai » 真行草の世界 (Le monde des formel, semi-formel, informel), in Nakamura Yasuo (dir.), Nibon geinōshi - dai 3 kan - chūsei 日本芸能史 - 第 3 巻 - 中世, Tōkyō, Hōsei daigaku shuppankyoku 法政大学出版局, 1983.

Nishiyama Matsunosuke 西山松之助, Watanabe Ichirō 渡辺一郎, Gunji Masakatsu 郡司正勝, Kinsei geidōron 近世芸道論 (La théorie de la voie de l'art à l'époque prémoderne), Tōkyō, Iwanami shoten 岩波書店, 1972.

Oмоте Akira 表章, ITō Masayoshi 伊藤正義, Konparu kodensho shūsei 金春古 伝書集成 (Recueil des anciens traités de Konparu), Tōkyō, Wan.ya shoten わ んや書店, 1969.

Oмоте Akira 表章, Amano Fumio 天野文雄, Iwanami kōza Nō kyōgen 岩波 講座 能・狂言 (Cours Iwanami, Nô et Kyōgen) I, Nögaku no rekishi能楽の 歴史 (Histoire du nô), Tōkyō, Iwanami shoten 岩波書占, 1987.

Омоте Akira 表章, ТАкемото Mikio 竹本幹夫, Iwanami kōza Nō kyōgen 岩 波講座 ・ 能 - 狂言 (Cours Iwanami, Nô et Kyōgen) II Nōgaku no densho to geiron 能楽の伝書と芸論 (Les traités et la théorie artistique du nô), Tōkyō, Iwanami shoten 岩波書店, 1988.

SHImizu Hiroshi 清水博, Seimeichi toshite no ba no ronri 生命知としての場 の論理 (La logique du champ comme connaissance de la vie), Tōkyō, Chūkō shinsho 中公新書, 1996.

Yagyū Munenori, trad. Nickels-Grolier Josette, Le Sabre de vie - Les enseignements secrets de la maison du Shôgun, Noisy-sur-École, Budo Éditions, 2005. 
Yokomichi Mario 横道萬里雄, Iwanami kōza Nō kyōgen 岩波講座 能・狂 言 (Cours Iwanami, Nô et Kyōgen) IV Nō no kōzō to gihō 能の構造と技法 (La structure et la technique du nô), Tōkyō, Iwanami shoten 岩波書店, 1987.

ZeAmi 世阿弥, Zenchiku 禅竹, compilé par Omote Akira 表章, KATō Shūichi 加藤周一, Zeami Zenchiku 世阿弥・禅竹 (Zeami et Zenchiku), Tōkyō, Iwanami shoten 岩波書店, 1974.

Résumé : On a souvent mis en avant l'importance du bouddhisme zen dans la constitution des techniques guerrières comme les arts martiaux, et en particulier l'influence du moine Takuan Sōhō sur Yagyū Munenori. La vision de l'identité du sabre et du zen (ken zen ichinyo) a été affirmée par ce moine dans le Fudōchi shinmyōroku (La sagesse immuable), un traité philosophique sur l'art du sabre qui mettait notamment l'accent sur la nécessité de garder l'esprit libre de tout attachement et de toute fixation. Mais on peut signaler également l'influence du théâtre nô, qui s'est rapproché de l'escrime notamment aux débuts de l'époque d'Edo à travers le lien intime entre les familles Yagyū et Konparu, une école du nô. Quel pourrait être alors l'apport du nô dans la constitution des arts guerriers ? Cet article éclaire les deux aspects de cette contribution : l'aspect conceptuel et l'aspect pratique.

Abstract: The importance of Zen Buddhism has often been emphasized in the constitution of warlike techniques such as martial arts, and in particular the influence of the monk Takuan Sōho on Yagyü Munenori. The vision of the identity of saber and zen (ken zen ichinyo) was stated by this monk in the Fudōchi shinmyōroku (Immutable Wisdom), a philosophical treatise on the art of the sword, which emphasized the need to keep the mind free from all attachment and fixation. But we can also point out the influence of Noh theater, which came close to fencing, especially at the beginning of the Edo period, through the intimate bond between the Yagyu and Konparu families, the latter a Noh school. What then could be the contribution of the Noh to the constitution of the warlike arts? This article sheds light on both aspects of this contribution: the conceptual and practical aspects. 


\section{CIPANGO}

258 Cahiers d'études japonaises n²2

Mots-clés : Nô, escrime, famille Konparu, famille Yagyū, bouddhisme zen, Takuan Sōhō, Yagyū Munenori

Keywords: Noh, the art of the sword, Konparu family, Yagyu family, Zen Buddhism, Takuan Sōhō, Yagyū Munenori

キーワード:能 剣術 金春家 柳生家禅 沢庵宗彭柳生宗矩 